\title{
Pleomorphic Xanthoastrocytoma of Childhood: MR Imaging and Diffusion MR Imaging Features
}

\author{
W. Moore, D. Mathis, L. Gargan, D.C. Bowers, L.J. Klesse, L. Margraf, and K. Koral
}

\begin{abstract}
BACKGROUND AND PURPOSE: Pleomorphic xanthoastrocytomas are rare astrocytic neoplasms of childhood and young adulthood. The purpose of this retrospective review was to evaluate MR imaging features of pediatric pleomorphic xanthoastrocytomas with an emphasis on diffusion MR imaging.
\end{abstract}

MATERIALS AND METHODS: Review of the neuro-oncology data base revealed 11 pediatric patients (range, 4.7-16.1 years) with pleomorphic xanthoastroacytomas with 9 of these patients having preoperative MR imaging available. Six patients had preoperative diffusion MR imaging. Demographics, histopathology slides, conventional imaging characteristics (location, cystic component, hemorrhage, enhancement, vasogenic edema, inner table scalloping), and ADC metrics (mean tumor ADC and tumor to normal brain ADC ratio) were evaluated.

RESULTS: Three pleomorphic xanthoastrocytomas had anaplastic features. Ten tumors were supratentorial. Two-thirds (6 of 9) of all tumors were either predominantly cystic or had cystic components, and three-fourths (6 of 8) of the supratentorial tumors had associated inner table scalloping. Seven of the 9 tumors had marked vasogenic edema ( $>10 \mathrm{~mm})$. Mean tumoral ADC $(n=7)$ was $912 \pm 219 \times 10^{-6}$ $\mathrm{mm}^{2} / \mathrm{s}$ (min-max: 617-1189). The tumor to normal brain ADC ratio was $1.14 \pm 0.26$ (min-max: 0.75-1.47).

CONCLUSIONS: Pleomorphic xanthoastrocytoma should be entertained in the differential diagnosis of peripheral supratentorial tumors that appear during childhood. Cysts, inner table scalloping, and marked vasogenic edema are relatively frequent features. Relatively low $A D C$ values and $A D C$ ratios are not uncommon in pleomorphic xanthoastrocytoma.

ABBREVIATION: PXA = pleomorphic xanthoastrocytoma

$\mathbf{P}$ leomorphic xanthoastrocytomas (PXAs) are rare neoplasms comprising less than $1 \%$ of all astrocytic tumors. ${ }^{1}$ They are more frequently encountered in childhood and young adulthood. ${ }^{2}$ Although there is relatively abundant literature on the histopathology and treatment of these tumors, there are fewer reports describing the imaging characteristics of PXAs. Moreover, imaging reports dedicated solely to pediatric PXAs are rare. The histopathologic descriptor "pleomorphic" aptly applies to the radiology of PXAs as well, resulting in myriad imaging presentations, potentially complicating the preoperative diagnosis. In the past several years, we encountered PXAs displaying hemorrhage and cysts, associated with marked vasogenic edema, and solid

Received March 9, 2014; accepted after revision April 8.

From the Departments of Radiology (W.M., K.K.), Pathology (D.M., L.M.), and Pediatrics (D.C.B., L.J.K.), University of Texas Southwestern Medical Center, Dallas, Texas; and Departments of Radiology (K.K.), Pathology (L.M.), Pediatrics (D.C.B., L.J.K.), and Neuro-Oncology (L.G.), Children's Medical Center, Dallas, Texas.

Please address correspondence to Korgun Koral, MD, Department of Radiology, University of Texas Southwestern Medical Center/Children's Medical Center, 1935 Medical District Dr, Dallas, TX 75235; e-mail: korgun.koral@utsouthwestern.edu

http://dx.doi.org/10.3174/ajnr.A4011 components showing relatively decreased water diffusion. These observations led to unnecessary inclusion of high-grade glial and embyronal tumors in differential diagnosis. Primitive neuroectodermal tumors, atypical teratoid rhabdoid tumors, and glioblastomas may present with imaging characteristics similar to those of PXAs. ${ }^{3,4}$ The purpose of this retrospective study was to seek MR imaging and diffusion MR imaging features that may be helpful in correctly diagnosing pediatric PXA preoperatively.

\section{MATERIALS AND METHODS}

The Institutional Review Board of the University of Texas Southwestern Medical Center approved this retrospective study and waived the requirement for informed consent. Review of the neurooncology database at Children's Medical Center revealed 11 pediatric PXAs diagnosed between January 1998 and April 2012. The imaging, pathology, and medical records of these patients were reviewed.

Histopathologic diagnoses were confirmed according to the 2007 World Health Organization classification of central nervous system tumors by a pediatric pathologist with $>20$ years experience (L.M.) and by a neuropathology fellow (D.M.). 
Individual patient information on demographics, histology, treatment, and outcome

\begin{tabular}{|c|c|c|c|c|c|c|c|c|c|}
\hline Patient No. & $\begin{array}{l}\text { Age (y)/ } \\
\text { Sex }\end{array}$ & Diagnosis & $\begin{array}{c}\text { ADC Value } \\
\left(\times 10^{-6} \mathrm{~mm}^{2} / \mathrm{s}\right)\end{array}$ & ADC Ratio & Treatment & $F / U(y)$ & $\begin{array}{l}\text { Treatment } \\
\text { Response }\end{array}$ & TTP (y) & F/U Results \\
\hline 1 & $4.7 / \mathrm{F}$ & PXA & & & XRT/Chemo & 14.8 & PD & 8.6 & Alive \\
\hline 2 & $4.8 / \mathrm{M}$ & PXA & 838 & 1.06 & Sx (3)/XRT & 5.0 & PD & 2.7 & Alive \\
\hline 3 & 7.1/F & Anap. PXA & & & Sx (2)/XRT/Chemo & 0.5 & PD & 0.2 & Died \\
\hline 4 & $9.3 / \mathrm{F}$ & PXA & 928 & 1.24 & Sx & 7.7 & $C R$ & $\mathrm{n} / \mathrm{a}$ & Alive \\
\hline 5 & $4.5 / \mathrm{F}$ & PXA & 668 & 0.85 & Sx & Lost to $\mathrm{F} / \mathrm{U}$ & & & \\
\hline 6 & $6.9 / \mathrm{M}$ & PXA & 1189 & 1.47 & Sx (3) & 9.6 & PD & 3.8 & Alive \\
\hline 7 & 16.1/M & PXA & 889 & 1.28 & Sx & 3.4 & $C R$ & $\mathrm{n} / \mathrm{a}$ & Alive \\
\hline 8 & $6.1 / \mathrm{M}$ & Anap. PXA & 825 & 0.75 & Sx/XRT/Chemo & 4.0 & $C R$ & $n / a$ & Alive \\
\hline 9 & $11.6 / \mathrm{M}$ & PXA & 762 & 1.32 & Sx & Lost to $\mathrm{F} / \mathrm{U}$ & & & \\
\hline 10 & $14.0 / \mathrm{F}$ & PXA & & & Sx/XRT/Chemo & 8.2 & PD & 0.8 & Died \\
\hline 11 & $13.0 / \mathrm{M}$ & Anap. PXA & & & Sx/XRT/Chemo & 2.5 & $C R$ & $\mathrm{n} / \mathrm{a}$ & Alive \\
\hline
\end{tabular}

Note:-Anap. indicates anaplastic; Chemo, chemotherapy; CR, complete remission; F/U, follow-up; PD, progressive disease; Sx, surgery; XRT, radiotherapy.

Of the 11 included patients, 9 had preoperative MR imaging available. Eight patients underwent scanning at our institution where $1.5 \mathrm{~T}$ and 3T scanners were used (Philips Healthcare, Best, the Netherlands). Conventional sequences included T1-weighted spin-echo (at least 2 orthogonal planes), FLAIR, T2-weighted spin-echo (coronal and axial), and gadolinium-enhanced 3 plane T1-weighted spin-echo. Diffusion MR imaging, performed using a spin-echo sequence with $b=0$ and $b=1000 \mathrm{~seconds} / \mathrm{mm}^{2}$, was available in 7 patients. ADC maps were generated with a monoexponential fit on a voxel-to-voxel basis for all imaging planes. Preoperative head CT was available in 4 patients.

Imaging studies were reviewed by a fourth year radiology resident (W.M.) and a pediatric neuroradiologist with 11 years of experience (K.K.) Tumor location, volume, and enhancement characteristics, presence of cyst(s), and presence/size of perilesional edema were evaluated in consensus. "Predominantly cystic" was defined as a solitary cyst and a smaller solid component and "multiple cysts" description was used if the tumors had more than 1 cystic component. When diffusion MR imaging was available, the solid components of the tumors were sampled by placing as many as 3 nonoverlapping ROIs on the ADC map. If tumors were large enough, 3 ROIs were used to sample the tumor more reliably. In 3 tumors with smaller solid components, only 1 region of interest could be placed on the tumor. A single ROI was placed on the normal contralateral thalamus because the thalamus provides a reliable internal control as it maintains its normal signal intensity on diffusion MR imaging even in cases of marked hydrocephalus. ${ }^{5}$ Mean tumor ADC values and tumor to normal brain ADC ratios were calculated. The head CT examinations were assessed for calcifications and hemorrhage.

\section{RESULTS}

\section{Patient Population}

The mean age at diagnosis was 8.9 years of age (range, 4.7-16.1 years). Six (54.5\%) patients were male and 5 (45.5\%) were female. Three of 11 tumors (27.3\%) demonstrated anaplastic features, increased mitotic activity, and often areas of necrosis. Ten of the $11(90.9 \%)$ tumors were supratentorial. The mean follow-up was 5.1 years (range, 0.02-14.8 years). Five of 11 (45.5\%) patients had evidence of recurrence or progression during follow-up. The mean time to progression was 3.2 years (range, $0.2-8.6$ years). The 5-year progression-free survival rate was $49.2 \%$. Two patients died, yielding an overall survival of $81.8 \%$ to date and a 5 -year survival rate of $88.9 \%$.

\section{Imaging}

CT and MR Imaging. No calcifications were identified in the 4 PXAs where CT was available. Nine patients had preoperative MR imaging available for review. The only infratentorial PXA predominantly involved the pons and to a lesser extent, the midbrain and medulla oblongata. The preoperative tumor volumes varied from 1.73 to $249.9 \mathrm{~cm}^{3}$ (mean $\left.=88.3, \mathrm{SD}=101.6\right)$. On the preoperative examinations, 8 of $9(88.9 \%)$ demonstrated intense heterogeneous enhancement of the solid component (all except the smallest tumor). Six of the $9(66.7 \%)$ tumors were either predominantly cystic or had multiple cysts. Six of the 8 (75\%) supratentorial tumors demonstrated scalloping of the inner table of the calvarium. Seven of the 9 (77.7\%) patients had significant vasogenic edema (defined as $\geq 10$ mm diameter). Three of 9 (33.3\%) tumors demonstrated hemorrhage.

Diffusion MR Imaging. Tumoral ADC values were measured in 7 patients. The mean tumoral ADC value was $912 \pm 219 \times 10^{-6}$ $\mathrm{mm}^{2} / \mathrm{s}$ (min-max: 617-1189). The tumor to normal brain ADC ratio was $1.14 \pm 0.26$ (min-max: $0.75-1.47)$. In the 7 patients who had preoperative diffusion MR imaging available, ADC ratios could not be used to determine the recurrence or survival. Of the 6 patients without anaplastic features, 4 underwent gross total resection and did not show recurrence (Table and Fig 1). The only patient with a PXA without anaplastic features and $\mathrm{ADC}$ ratio $<1$ underwent gross total resection, but was lost to follow-up. One patient had a hippocampal and parahippocampal gyrus tumor (ADC ratio, 1.47) and underwent subtotal resections and had a progressive tumor. One patient (ADC ratio, 1.06) had progressive disease about the left middle cerebral artery after subtotal resection and underwent radiation therapy and chemotherapy (Fig 2). The only patient with PXA with anaplastic features in whom preoperative diffusion MR imaging was available (ADC ratio, 0.75) underwent gross total resection, chemotherapy, and radiation therapy (Fig 3). This patient had no signs of recurrence at 4-year follow-up.

\section{DISCUSSION}

In this study, we report preoperative MR imaging findings of pediatric PXAs and describe the diffusion MR imaging metrics of these rare tumors. Consistent with the histopathologic descriptor "pleomorphic," the imaging features of PXAs may be varied and generate some difficulty in the preoperative diagnosis. Although the mean age at presentation is 26 years, PXAs can be encountered in all age groups ranging from infancy to the ninth decade. ${ }^{2,6}$ PXAs are overwhelm- 

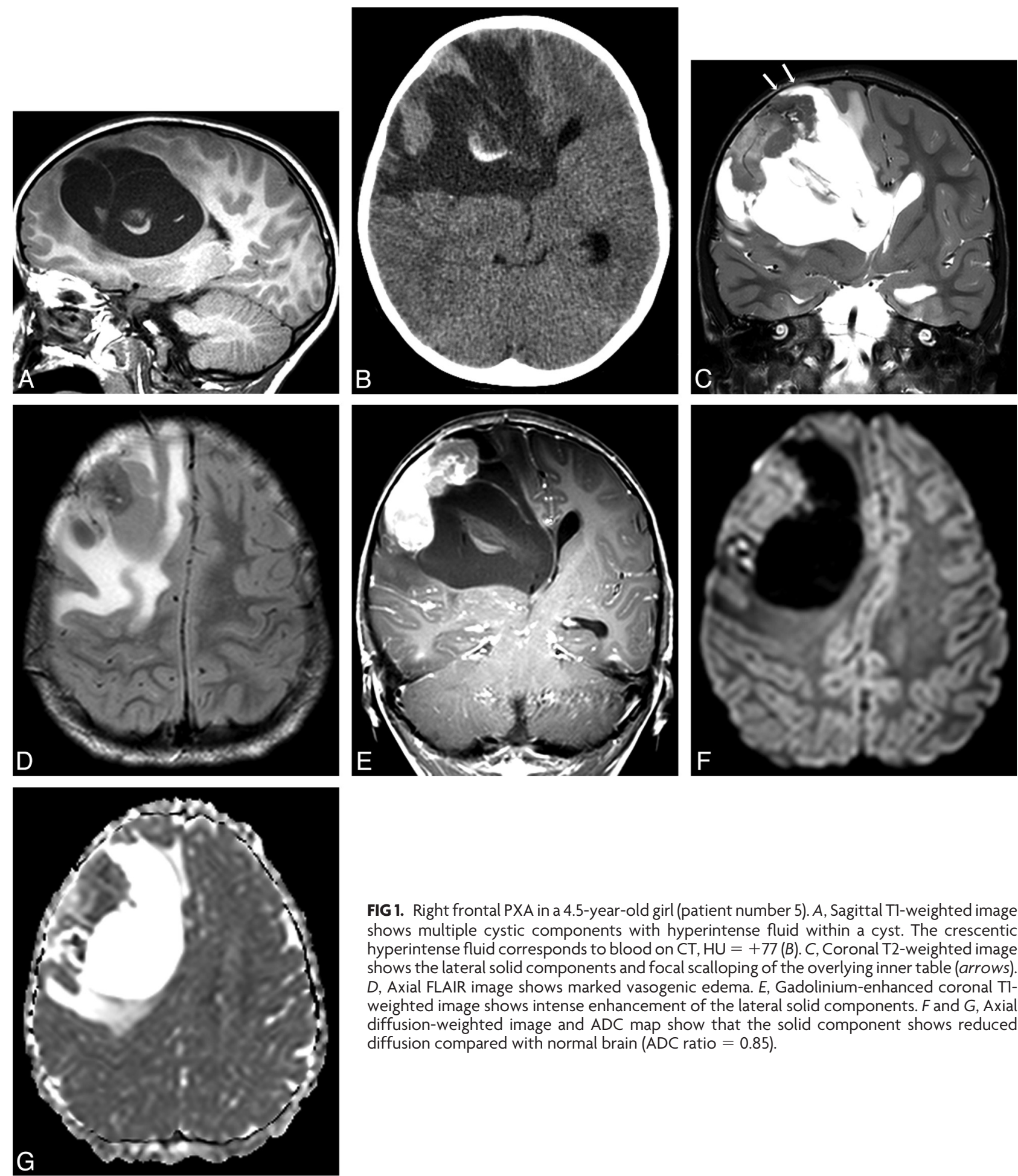

FIG 1. Right frontal PXA in a 4.5-year-old girl (patient number 5). A, Sagittal T1-weighted image shows multiple cystic components with hyperintense fluid within a cyst. The crescentic hyperintense fluid corresponds to blood on $\mathrm{CT}, \mathrm{HU}=+77(B)$. C, Coronal T2-weighted image shows the lateral solid components and focal scalloping of the overlying inner table (arrows). $D$, Axial FLAIR image shows marked vasogenic edema. E, Gadolinium-enhanced coronal T1weighted image shows intense enhancement of the lateral solid components. $F$ and $G$, Axial diffusion-weighted image and ADC map show that the solid component shows reduced diffusion compared with normal brain (ADC ratio $=0.85$ ).

ingly supratentorial tumors (99\%); in a study based upon the National Cancer Institute's Surveillance, Epidemiology, and End Results database, ${ }^{6}$ only 2 cerebellar tumors were present among 214 PXAs. The only infratentorial PXA in our series was a brain stem lesion without involvement of the cerebellum. PXAs tend to be superficial in the cerebral hemispheres and $40 \%-50 \%$ involve the temporal lobes. ${ }^{2,6}$ In the largest series where presenting symptoms were known, $71 \%$ of the patients' presenting symptoms were seizures only, which were often of long duration (median 3 years). ${ }^{2}$

According to the latest World Health Organization classification,
PXAs represent grade II tumors histologically. ${ }^{2}$ A small percentage of PXAs demonstrate increased mitotic activity (defined as 5 or more mitoses per 10 high-power fields) with or without areas of necrosis and are designated as "PXA with anaplastic features." ${ }^{2}$ PXAs are remarkable for a relatively favorable outcome with 5- and 10-year overall survival rates of $75 \%-81 \%$ and $67 \%-70 \%$, respectively. ${ }^{2,6}$ The survival rates in our cohort are comparable. Gross total resection of the tumor and a low mitotic rate were found to correlate positively with overall survival. ${ }^{2}$ Although PXAs are generally considered indolent neoplasms, they are associated with a higher frequency of recur- 

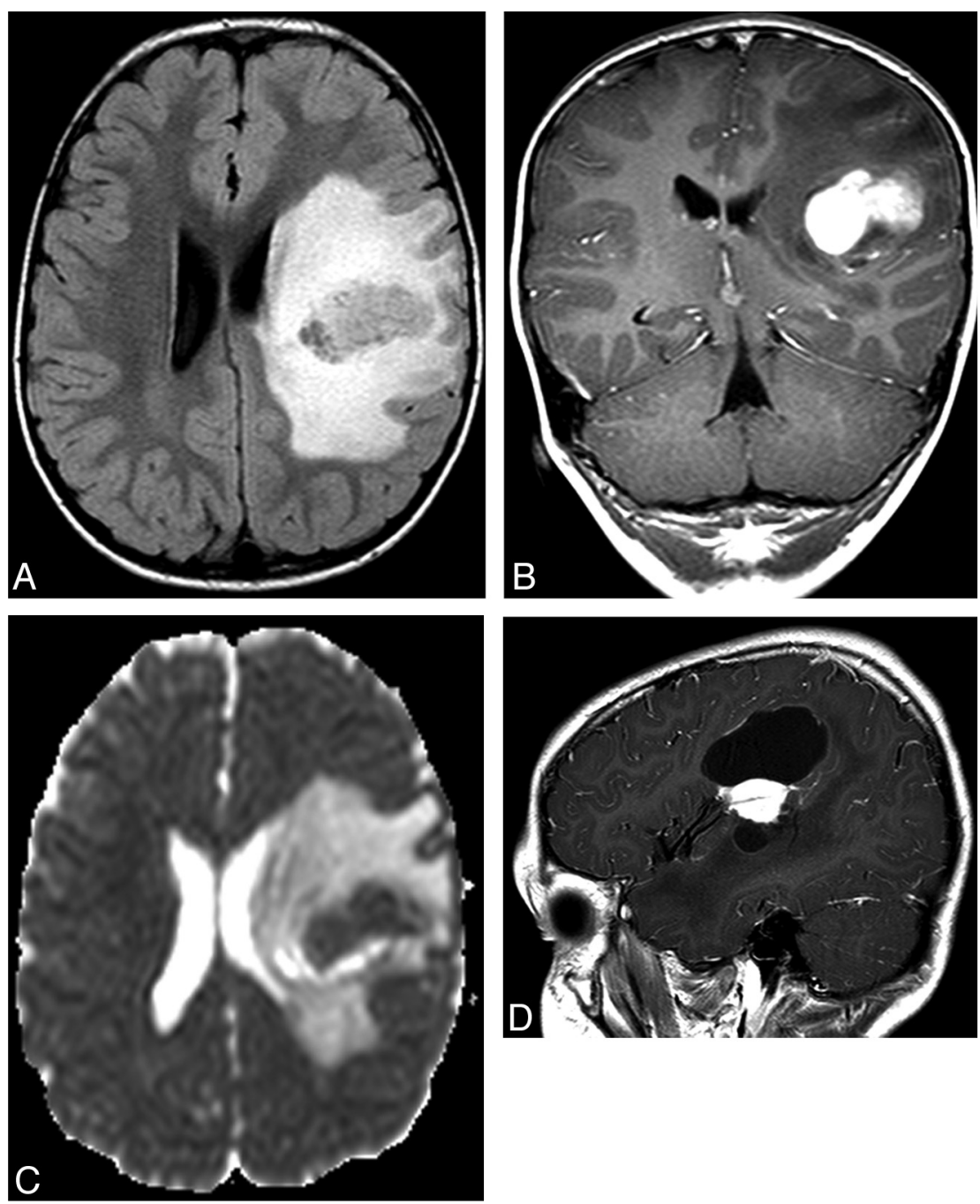

FIG 2. Left frontal PXA in a 4.8-year-old boy (patient number 2). A, Preoperative axial FLAIR image shows marked vasogenic edema. $B$, Preoperative coronal T1-weighted gadolinium-enhanced image shows intense enhancement of the lesion. $C$, The calculated preoperative ADC ratio was 1.06.D, The mass could not be resected completely because of its proximity to the left middle cerebral artery and showed progression over 4 years. Sagittal gadolinium-enhanced T-weighted image shows the signal void of a middle cerebral artery branch within the enhancing mass.

rence, malignant transformation, and death, compared with other low-grade gliomas, such as pilocytic astrocytomas. ${ }^{6-9}$

Although there are imaging reports of PXAs in children and adults, dedicated pediatric imaging studies are rare and diffusion MR imaging characteristics were not previously described. ${ }^{10-14}$ In 1 of the earliest MR imaging reports, Lipper et $\mathrm{al}^{12}$ described imaging findings of PXA in 7 patients, 3 of whom were children. Peripheral location of PXA in the cerebral hemispheres was emphasized in this report. We identified 3 studies with $>15$ patients where imaging findings were described in detail. ${ }^{10,15,16}$ Crespo-Rodríguez et $\mathrm{al}^{10}$ described preoperative MR imaging findings in 10 children (mean age $=11.2$ years, range $=9-14$ years) in a publication of pediatric and adult PXAs. Eight of these tumors were cystic with a mural nodule; this feature was recognized with similar frequency in the adult population of the same study. In a dedicated pediatric PXA report, 8 of the 11 tumors had an enhancing mass with a cystic component. ${ }^{8}$ Our cohort shows similarities with these reports with two-thirds of tumors being predominantly cystic or containing multiple cysts.

In our study, we found that 6 of the 8 supratentorial tumors showed inner table scalloping. Inner table scalloping is a feature of slow-growing superficial brain tumors of childhood, such as ganglioglioma and dysembryoplastic neuroepithelial tumor. ${ }^{17}$ Whereas Crespo-Rodríguez et a ${ }^{10}$ reported inner table remodeling in 3 of 6 patients with PXA younger than 12 years, in the study by $\mathrm{Yu}$ et al, ${ }^{16}$ only 1 of the 19 patients (both children and adults with an age range of 8-70 years) demonstrated calvarial remodeling.

Our findings are similar to the previously published reports in the frequency of perilesional edema; however, we recognized that marked perilesional edema, described as $>10 \mathrm{~mm}$, was present in 7 of our 9 PXAs. Lipper et $\mathrm{al}^{12}$ reported "definite" perilesional edema (mild or moderate) in 5 of 7 cases. In the study of Crespo-Rodríguez et $\mathrm{al},{ }^{10}$ perilesional edema was present in 2 of the 10 pediatric patients, similar to the frequency seen in their adult patients with PXA. In a more recent study that reported perilesional edema in greater detail, $31.8 \%$ (7 of 22) of the patients had edema $>10 \mathrm{~mm}$, while $36.4 \%(8 / 22)$ had edema $<10 \mathrm{~mm} \cdot{ }^{15}$ In this study, all of the 9 pediatric PXAs showed perilesional edema; in $7 \mathrm{pa}-$ tients, edema was $<10 \mathrm{~mm}$, and in 2 it was $>10 \mathrm{~mm}^{.15}$

Hemorrhage in PXA has been described as an uncommon im- 

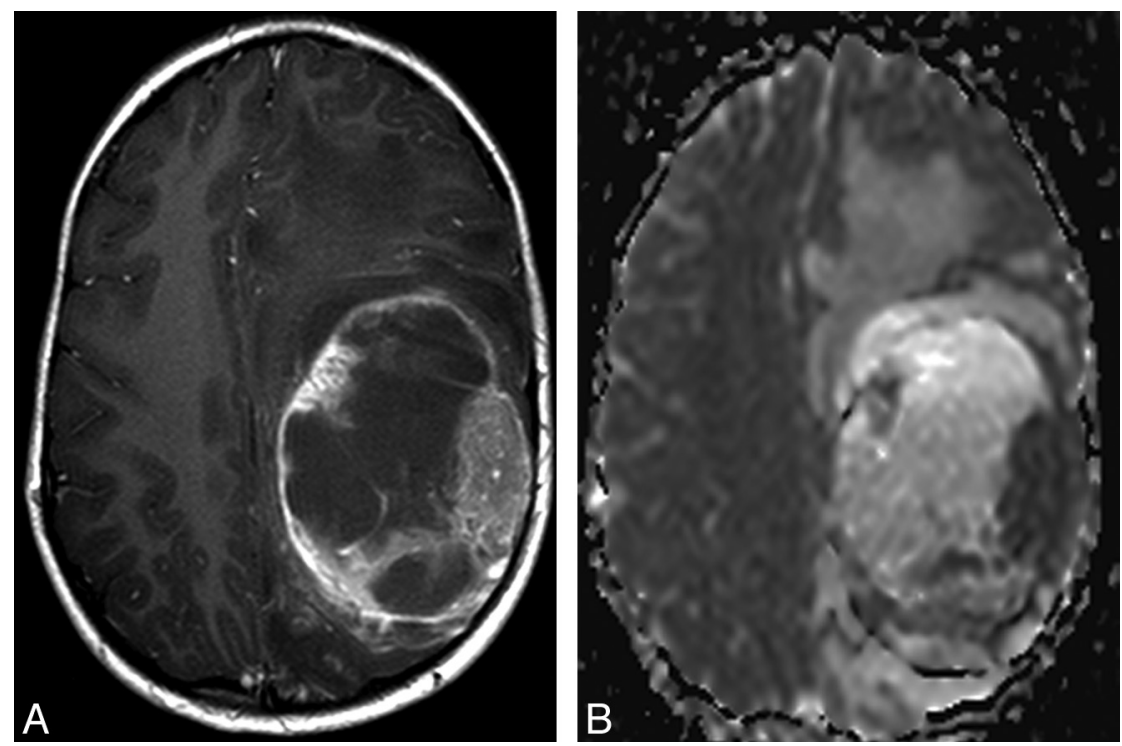

white matter appears to be a common observation. Relatively lower ADC values and $\mathrm{ADC}$ ratios of the solid components may distinguish these tumors from other lowgrade supratentorial, peripheral tumors such as gangliogliomas.

\section{REFERENCES}

1. Giannini C, Paulus W, Louis DN, et al. Pleomorphic xanthoastrocytoma. In: Louis DN, Ohgaki H, Wiestler OD, et al, eds. WHO Classification of Tumours of the Central Nervous System. 4th ed. Lyon, France: International Agency for Research on Cancer; 2007:22-24

2. Giannini C, Scheithauer BW, Burger PC, et al. Pleomorphic xanthoastrocytoma: what do we really know about it? Cancer 1999;85:2033-45

3. Borja MJ, Plaza MJ, Altman N, et al. Conventional and advanced MRI features of pediatric intracranial tumors: supratentorial tumors. AJR Am J Roentgenol 2013;200:W483-503

4. Chang YW, Yoon HK, Shin HJ, et al. MR imaging of glioblastoma in children: usefulness of diffusion/perfusion-weighted MR and MR spectroscopy. Pediatr Radiol 2003;33:836-42

5. Gimi B, Cederberg K, Derinkuyu B, et al. Utility of apparent diffusion coefficient ratios in distinguishing common pediatric cerebellar tumors. Acad Radiol 2012;19:794-800

6. Perkins SM, Mitra N, Fei W, et al. Patterns of care and outcomes of patients with pleomorphic xanthoastrocytoma: a SEER analysis. J Neurooncol 2012;110:99-104

7. Marton E, Feletti A, Orvieto E, et al. Malignant progression in pleomorphic xanthoastrocytoma: personal experience and review of the literature. J Neurol Sci 2007;252:144-53

8. Rao AA, Laack NN, Giannini C, et al. Pleomorphic xanthoastrocytoma in children and adolescents. Pediatr Blood Cancer 2010;55:290-94

9. Tekkok IH, Sav A. Anaplastic pleomorphic xanthoastrocytomas. Review of the literature with reference to malignancy potential. $\mathrm{Pe}$ diatr Neurosurg 2004;40:171-81

10. Crespo-Rodríguez AM, Smirniotopoulos JG, Rushing EJ. MRI and CT imaging of 24 pleomorphic xanthoastrocytomas (PXA) and a review of the literature. Neuroradiology 2007;49:307-15

11. Goncalves VT, Reis F, Queiroz Lde S, et al. Pleomorphic xanthoastrocytoma: magnetic resonance imaging findings in a series of cases with histopathological confirmation. Arq Neuropsiquiatr 2013;71:35-39

12. Lipper MH, Eberhard DA, Phillips CD, et al. Pleomorphic xanthoastrocytoma, a distinctive astroglial tumor: neuroradiologic and pathologic features. AJNR Am J Neuroradiol 1993;14:1397-404

13. Tien RD, Cardenas CA, Rajagopalan S. Pleomorphic xanthoastrocytoma of the brain: MRI findings in six patients. AJR Am J Roentgenol 1992;159:1287-90

14. Wallace DJ, Byrne RW, Ruban D, et al. Temporal lobe pleomorphic xanthoastrocytoma and chronic epilepsy: long-term surgical outcomes. Clin Neurol Neurosurg 2011;113:918-22

15. Lim S, Kim JH, Kim SA, et al. Prognostic factors and therapeutic outcomes in 22 patients with pleomorphic xanthoastrocytoma. $J$ Korean Neurosurg Soc 2013;53:281-87

In this study we reported preoperative imaging findings of pediatric PXAs with emphasis on diffusion MR imaging. PXA should be entertained in the differential diagnosis of peripheral cerebral hemispheric lesions with solid and cystic components associated with scalloping of the adjacent inner table. Vasogenic edema of the adjacent

16. Yu S, He L, Zhuang X, et al. Pleomorphic xanthoastrocytoma: MR imaging findings in 19 patients. Acta Radiol 2011;52:223-28

17. Shin JH, Lee HK, Khang SK, et al. Neuronal tumors of the central nervous system: radiologic findings and pathologic correlation. Radiographics 2002;22:1177-89 